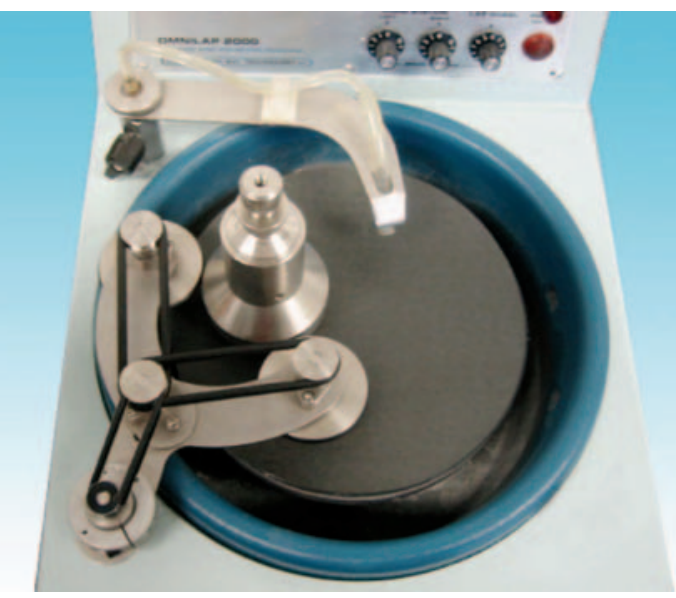

FIG.1. Polishing setup used for the preparation process.

strain induced from ion beam bombardment [5].

A simple approach of mechanical polishing utilizing standard metallographic processes has been used on a wide variety of materials to produce EBSD quality samples. The technique uses a semi-automatic polishing machine combined with a precision lapping fixture to control the polishing process. Thickness control of each step is critical in the ability to eliminate mechanical deformation of the sample and to ensure that a high quality EBSD pattern will be obtained. Metal samples of unknown compositions of brass, stainless steel, and copper were prepared using a Model 920 Lapping and Polishing System combined with a Model 150 Lapping and Polishing fixture. A series of silicon carbide abrasive

\section{High Pressure Freezing User Group Meeting Launched at M \& M 2004, More to Come}

The nation's first High-Pressure Freezing (HPF) User Group Meeting, hosted by by BAL-TEC RMC, a joint marketing alliance comprised of BAL-TEC AG, Boeckeler RMC Products, and TechnoTrade International, Inc., was successfully launched during M \& M 2004 on Sunday, August 1, in Savannah, Ga. About 40 attendees came from all over the U.S., ranging in disciplines from plant pathology, biology, and neuroscience, to chemistry and pharmaceuticals. All have either used or plan to use high-pressure freezing as a method to freeze specimens intended for examination in the electron microscope (EM).

High-pressure freezing is especially used for freezing large tissue specimens without requiring structure-altering freeze protective additives. It is ideally suited for use in conjunction with subsequent freeze substitution, followed by low temperature embedding and polymerization for sectioning in a conventional ultramicrotome for morphological preservation and immunogold labeling. HPF is also well suited for subsequent replication by freeze fracturing in a freeze etching system for transmission electron microscopy (TEM), subsequent cryosectioning for cryoTEM and cryo scanning electron microscopy (SEM) investigations.

\section{Summary of Presentations}

Presenters for the meeting included Robert Apkarian of Emory University in Georgia, who showed how HPF is used in imaging self-assembled organic hydrogels by high-resolution cryoSEM. Allison Vendemeene of Arizona State University, a post-doc in Robbie Roberson's lab, showed how superior morphologic preservation of yeast for TEM tomography could be accomplished with HPF. Her images detailed the improved morphology of samples difficult to preserve with traditional chemical fixatives.
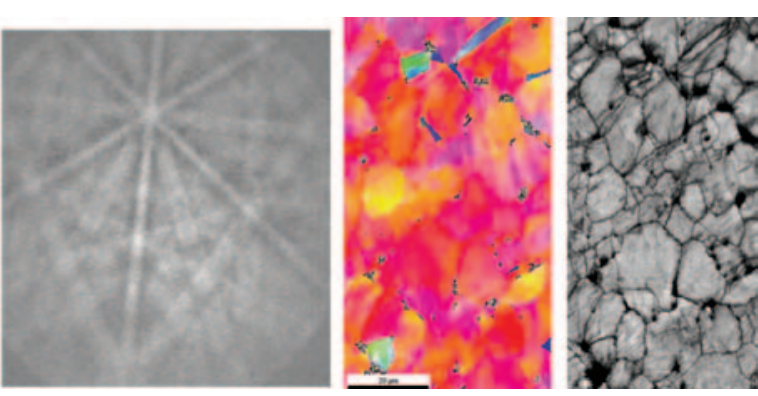

FIG. 2. A) Surface image of the brass sample taken using SEM, B) EBSD pattern acquired from the sample; C) Image quality map of the polished sample demonstrating a

papers from $240,400,600,800,1000$, and 1200 grits were used for preparation, each taking 5-10 minutes per step. Final polishing was done using $1 \mu \mathrm{m}$ diamond suspension on Satin cloth followed by $0.05 \mu \mathrm{m}$ colloidal silica polishing on a MultiTex $\mathrm{x}^{\mathrm{mit}}$ polishing cloth for 5-10 minutes. The equipment setup is shown in Figure 1, with the results given in Figure 2. The image quality map shows a high quality surface finish that is deformation free, and the EBSD pattern is easily acquired using these preparation methods.

\section{References}

[1] http://www.edax.com

[2] J. R. Michael, MSA Proc., Vol. 7, Supp. 2, 2001, p. 380-381

[3] M. Wall, et al, MSA Proc., Vol 6, Supp. 2, 2000, p. 950-951

[4] R. Yoda, et al, MSA Proc., Vol 8, Supp. 2, 2002, p. 676-677

[5] J. K. Farrer, et al, MSA Proce., Vol 8, Supp. 2, 2002, p. 544-545

Maryann Martone of the University of California, San Diego, gave a popular presentation on how to use an XML-based database called NeuroSys to catalog and manage large data sets of HPF information.

Roger Wepf of Beiersdorf, AG, Germany, gave two presentations. His first was on closing the gap between histology and ultrastructure research by using 3D Correlative Microscopy with HPF. He explained the advantage to imaging one and the same sample by using HPF followed by a modified freeze substitution process. This allows investigation of the identical sample in the light microscope (histology), the confocal microscope (3D morphology) and the TEM (ultrastructure). The aim of this approach is to have $3 \mathrm{D}$ datasets from the identical structure at different resolution and content levels, a so-called multimodal image dataset for further structural investigations.

Wepf's second presentation discussed HPF for high-resolution cryo-SEM. Here, he showed the advantage of the different freezing techniques and compared the various structural data one can obtain by cryo-SEM.

David Hall of the Albert Einstein College of Medicine in New York, presented the advantages of low-temperature fixation for $C$. elegans by using HPF and freeze substitution techniques. HPF captures fast membrane events that are lost during slow chemical fixation. He also detailed freeze substitution protocols to preserve antigenic activity for immunogold labeling.

\section{The Next Meeting}

Plans are in the works to incorporate a regular HPF User Group meeting at future M \& M meetings. If all goes as planned, the next one will be held on Sunday, July 31, 2005 in Hawaii. HPF users or potential users who wish to be on the invitation list for the user group should contact Dave Roberts of Boeckeler Instruments, Inc., at (800) 552-2262 or e-mail him: dave@boeckeler.com. 


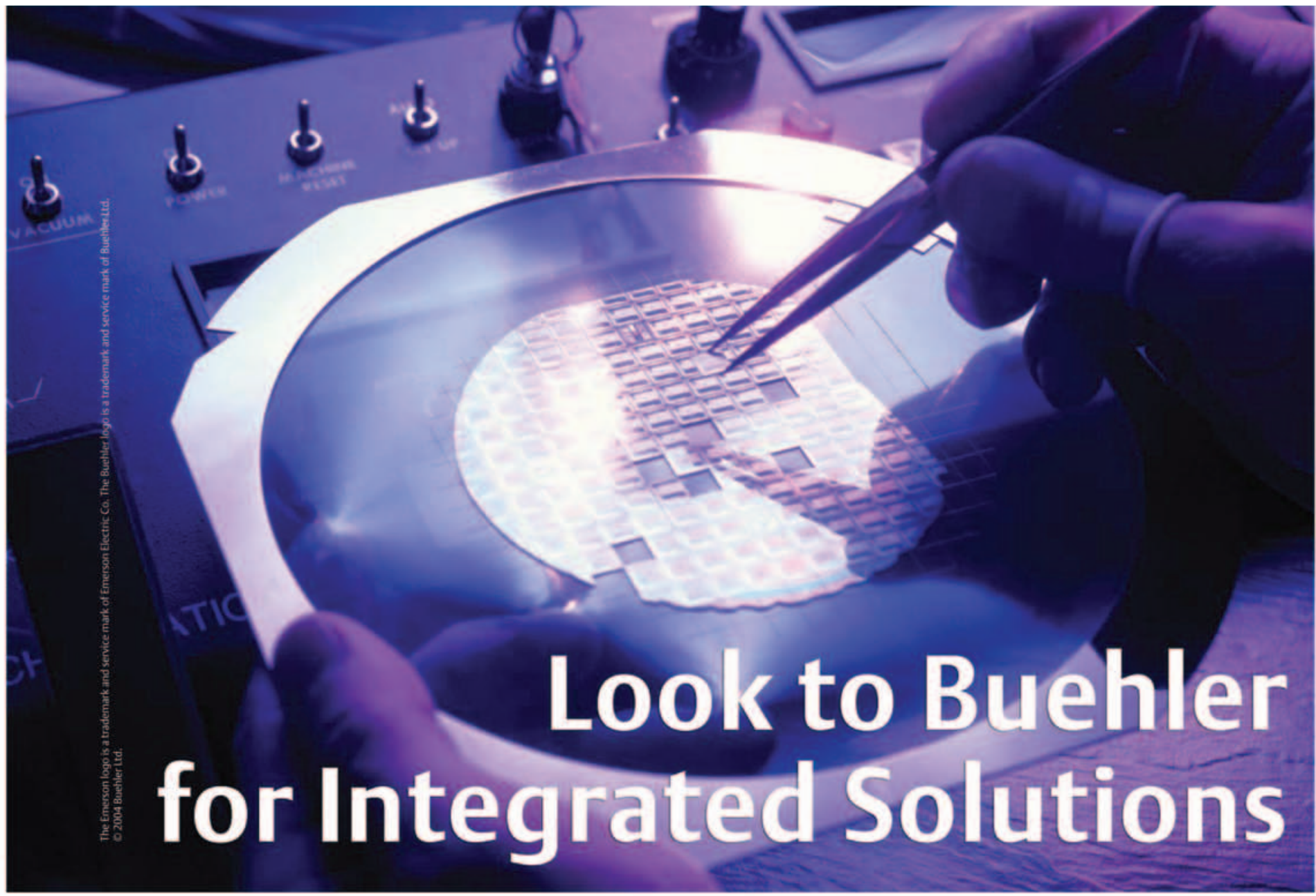

We know how important it is to make all the right connections, including finding a supplier who understands and meets your needs. As the premier company in our field since 1936, Buehler offers a complete family of preparation equipment and consumables combined with personal service.

Microscopic examination is important for electronic devices; and, Buehler offers the tools you need for superior results. The IsoMet ${ }^{\star} 5000$ Linear Precision Saw ensures you can section your specimen as close to the area of interest as possible, bypassing much of the grinding that consumes both abrasives and operator time. The addition of a Precision Table with $180^{\circ}$ rotation in $1^{\circ}$ increments is ideal for sectioning thin materials and wafers.

Consider the MPC" 2000 Integrated Circuit Cross-Sectioning System and the MPC' 3000 Integrated Circuit Backside Grinding System for further process improvements. These systems are specifically designed for electronic components to remove material to target depth, reduce the number of grinding steps and simplify microscopic inspection.

Our wide array of products and technical information makes Buehler your best connection! Think of All the Possibilities .... Because We Have A Million"

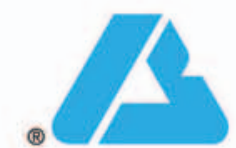

Uㅡ늩

Worldwide Headquarters Buehler Ltd • 41 Waukegan Road Lake Bluff, Illinois $60044 \cdot$ USA Tel: (847) 295-6500 Fax: (847) $295-7979$ Email: info@buehler.com Web Site: http://www.buehler.com (An Emerson Industrial Automotion subsidiary since 1992) 